Hardy-Ramanujan Joumal

Vol.16 (1993) 1-20

\title{
On sets of coprime integers in intervals
}

\section{P. Erdös and Sárközy ${ }^{1}$}

1. Throughout this paper we use the following notations : $\mathbb{Z}$ denctes the set of the integers. $N$ denotes the set of the positive integers. For $\mathcal{A} \subset N, m \in N, u \in \mathbb{Z}$ we write $\mathcal{A}_{(m, u)}=\{a: a \in \mathcal{A}, a \equiv u(\bmod m)\} \cdot \varphi(n)$ denotes Euler'o function. $p_{k}$ denotes the Lth $^{\text {th }}$ prime: $p_{1}=2, p_{2}=3, \cdots$ and we put $P_{k}=\prod_{i=1}^{k} p_{i}$. If $k \in N$ and $k \geq 2$, then $\Phi_{k}(\mathcal{A})$ denotes the number of the $k$-tuples $\left(a_{1}, \cdots, a_{k}\right)$ such that $a_{1} \in \mathcal{A}, \cdots, a_{k} \in \mathcal{A}, a_{1}<a_{2}<\cdots<a_{k}$ and $\left(a_{i}, a_{j}=1\right)$ for $1 \leq i<j \leq k$. If $k \in N, \mathcal{A} \subset N$ and $\Phi_{k}(\mathcal{A})=0$, i.e., $\mathcal{A}$ does not contain a subset $S$ consisting of $k$ pairwise coprime integers, then $\mathcal{A}$ is said to have property $\mathbf{P}_{k}$, and $\Gamma_{k}$ denotes the famity of those subsets of $N$ which have property $\mathbf{P}_{k}$. We write

$$
F_{k}(n)=\max _{\substack{A \subset\{i, \cdots, n\} \\ \mathcal{A} \in \Gamma_{k}}}|A|
$$

(In other words, $t=F_{k}(n)+1$ is the smallest positive integer such that every set $B$ with $B \subset\{1, \cdots, n\},|B|=t$ cuntains $k$ pairwise coprime integers). Moreover, for $k, m, n \in N$ we write

$$
g_{k}(m, n)=\max _{\mathcal{A} \subset\{m, m+1, \ldots, m+n-1\}}|\mathcal{A}|
$$

\footnotetext{
${ }^{3}$ Research patimliy supported by Hungarian Nationai Founciation for Srientife Research, Grant No. 1901.
} 
and

$$
G_{k}(n)=\max _{m \in N} g_{k}(m, n)
$$

so that, clearly,

$$
\left(g_{k}(1, n)=\right) F_{k}(n) \leq G_{k}(n) .
$$

For $k, m, n \in N$, let $\psi_{k}(m, n)$ denote the number of those integers $u \in$ $\{m, m+1, \cdots, m+n-1\}$ which are multiples of at least one of the first $k$ primes, and write

$$
\Psi_{k}(n)=\psi_{k}(1, n) \text {. }
$$

The set $\mathcal{A} \stackrel{\text { def }}{=}\left\{a: a \in\{m, m+1, \cdots, m+n-1\},\left(a, P_{k-1}\right)>1\right\}$ has property $P_{k}$ and thus for this set $\mathcal{A}$ we have

$$
g_{k}(m, n) \geq|\mathcal{A}|=\psi_{k-1}(m, n),
$$

in particular,

$$
g_{k}(1, n)=F_{k}(n) \geq \psi_{k-1}(1, n)=\Psi_{k-1}(n)
$$

Clearly for all $m, n \in N$ we have

$$
\begin{aligned}
& \psi_{k}(m, n)=\left|\left\{u: m \leq u<m+n,\left(u, P_{k}\right)>1\right\}\right|= \\
& =|\{u: m \leq u<m+n\}|-\sum_{d \mid P_{k}} \mu(d)|\{u: m \leq u<m+n, d \mid u\}|=
\end{aligned}
$$

(5)

$$
=n-\sum_{d \mid P_{k}} \mu(d)\left(\left[\frac{m+n}{d}\right]-\left[\frac{m}{d}\right]\right)
$$

whence

$$
\begin{aligned}
& \left|\psi_{k}(m, n)-\left(1-\prod_{i=1}^{k}\left(1-\frac{1}{p_{i}}\right)\right) n\right|= \\
& =\left|\left(n-\sum_{d \mid P_{k}} \mu(d)\left(\left[\frac{m+n}{d}\right]-\left[\frac{m}{d}\right]\right)\right)-\left(n-\sum_{d \mid \mathbb{P}_{k}} \mu(d) \frac{n}{d}\right)\right| \leq
\end{aligned}
$$


(6)

$$
\begin{aligned}
& \leq \sum_{d \mid P_{k}}\left|\mu(d) \|\left[\frac{m+n}{d}\right]-\left[\frac{m}{d}\right]-\frac{n}{d}\right| \leq \\
& \leq \sum_{d \mid P_{k}}\left(\left|\left[\frac{m+n}{d}\right]-\frac{m+n}{d}\right|+\left|\frac{m}{d}-\left[\frac{m}{d}\right]\right|\right)<2 \sum_{d \mid P_{k}} 1=2^{k+1} .
\end{aligned}
$$

If $P_{k} \mid n$, then we have $|\{d: m \leq u<m+n, d \mid u\}|=n / d$ thus it follows from (5) that

(7) $\quad \psi_{k}(m, n)=n-\sum_{d \mid P_{k}} \mu(d) \frac{n}{d}=\left(1-\prod_{i=1}^{k}\left(1-\frac{1}{p_{i}}\right)\right) n$ for $P_{k} \mid n$

In particular, we have

$$
\Psi_{k}(n)=\psi_{k}(1, n)=\left|\left\{u: u \in N, u \leq n,\left(u, P_{k}\right)>1\right\}\right|=
$$

$$
=-\sum_{d \mid P_{k}, d>1} \mu(d)\left[\frac{n}{d}\right] \text { for all } n \in N
$$

and

$$
\Psi_{k}(n)=\left(1-\prod_{i=1}^{k}\left(1-\frac{1}{p_{i}}\right)\right) n \text { for } n \in N, P_{k} \mid n .
$$

Finally, for $k, \ell, m, n \in N, h_{(k, \ell)}(m, n)$ denotes the maximum of the cardinalities of the sets $\mathcal{A}$ such that $\mathcal{A} \subset\{m, m+1, \cdots, m+n-1\},\left(a, P_{k}\right)=1$ for all $a \in \mathcal{A}$ and $\mathcal{A} \in \Gamma_{\ell}$.

2. It is easy to see that

$$
F_{2}(n)=\Psi_{1}(n)=\left[\frac{n}{2}\right]
$$

and

$$
F_{3}(n)=\Psi_{2}(n)=\left[\frac{n}{2}\right]+\left[\frac{n}{3}\right]-\left[\frac{n}{6}\right] \quad\left(=\frac{2}{3} \text { for } 6 \mid n\right) .
$$

Erdös, Sárközy and Szemerédi [3],[4] extended and sharpened these statements in various directions, Erdös conjectured long ago (see, e.g., [2]) that

$$
F_{k}(n)=\Psi_{k-1}(n)
$$


Szabó and Toth [5] proved this in the special case $k=4$ :

$$
F_{4}(n)=\Psi_{3}(n)
$$

However, the general case seems to be hopeless at the present.

In this paper, our goal is to study the case of general $k$ and to prove several partial results. In particular, in sections 3 and 4 we will study the connection between the functions $F_{k}(n)$ and $G_{k}(n)$. In section 5 , the function $h_{(k, \ell)}(m, n)$ will be studied. In section 6 , we will give an upper bound for $G_{k}(n)$. Finally, in section 7 , we will generalize several results proved in [3] and [4] by estimating $\Phi_{k}(\mathcal{A})$.

3. By (2), $F_{k}(n) \leq G_{k}(n)$ for all $n$. First we remark that for $k=2$ and 3 , both $<$ and $=$ occur infinitely often in this inequality.

THEOREM 1. If $m, u, v \in N$, then

(12) $g_{2}(m, 2 v)=v$,

(13) $g_{2}(2 u, 2 v+1)=v+1$,

(14) $g_{2}(2 u-1,2 v+1)=v$,

(15) $g_{3}(m, 6 v)=4 v$,

(16) $g_{3}(6 u-5,6 v-1)=4 v-1$,

(17) $g_{3}(6 u, 6 v-1)=\frac{1}{x} \ddot{v}$.

It follows trivially from Theorem 1 that

COROLLARY 1. For all $v \in N$ we have

$$
\begin{aligned}
& F_{2}(2 v)=G_{2}(2 v)=v \\
& G_{2}(2 v+1)=v+1=F_{2}(2 v+1)+1 \\
& F_{3}(6 v)=G_{3}(6 v)=4 v
\end{aligned}
$$

and 


$$
G_{3}(6 v-1) \geq 4 v=F_{3}(6 v-1)+1 .
$$

PROOF OF THEOREM 1. (12),(13) and (14) are near trivial, thus we prove only (15),(16) and (17). By (3) and (5), for all $m, n \in N$ we have

$$
g_{3}(m, n) \geq \psi_{2}(m, n)=N_{1}+N_{2}-N_{3}
$$

where

$$
\begin{aligned}
& N_{1}=|\{a: a \in\{m, m+1, \cdots, m+n-1\}, 2 \mid a\}| \\
& N_{2}=|\{a: a \in\{m, m+1, \cdots, m+n-1\}, 3 \mid a\}|
\end{aligned}
$$

and

$$
N_{3}=|\{a: a \in\{m, m+1, \cdots, m+n-1\}, 6 \mid a\}| .
$$

It is easy to see that

$$
N_{1}=3 v, N_{2}=2 v \text { and } N_{3}=v \text { for all } m \text { and } n=6 v
$$

(20) $N_{1}=3 v-1, N_{2}=2 v-1$ and $N_{3}=v-1$ for $m=6 u-5, n=6 v-1$

and

$$
N_{1}=3 v, N_{2}=2 v \text { and } N_{3}=v \text { for } m=6 u, n=6 v-1
$$

It follows from (18), (19), (20) and (21) that

$$
g_{3}(m, 6 v) \geq 6 v, g_{3}(6 u-5,6 v-1) \geq 4 v-1, g_{3}(6 u, 6 v-1) \geq 4 v .
$$

Now we will show that

$$
g_{3}(t, 6) \leq 4 \text { for all } t \in N
$$

In fact, if $\mathcal{A} \subset\{t, t+1, \cdots, t+5\}$ and $|\mathcal{A}| \geq 5$, then either $\mathcal{A}$ contains three consecutive odd numbers or $\mathcal{A}$ contains two consecutive odd numbers and an 
even number divisible by either 3 or 5 . In both cases, these three numbers are pairwise coprime so that $\mathcal{A}$ cannot have property $\mathrm{P}_{3}$ which proves (19). Clearly, for all $k, a, b, c \in N$ we have

$$
g_{k}(a, b+c) \leq g_{k}(a, b)+g_{k}(a+b, c) .
$$

By (23) and (24) we have

$$
g_{3}(m, 6 v) \leq \sum_{i=0}^{v-1} g_{3}(m+6 i, 6) \leq \sum_{i=0}^{v-1} 4=4 v .
$$

(15) follows from (22) and (25).

Next we will show that

$$
g_{3}(6 u-5,5) \leq 3 \text {. }
$$

In fact, if $\mathcal{A} \subset\{6 u-5,6 u-4,6 u-3,6 u-2,6 u-1\}$ and $|\mathcal{A}| \geq 4$, then either $\mathcal{A}$ contains three consecutive odd numbers or $\mathcal{A}$ contains $6 u-4,6 u-2$ and one of $6 u-5,6 u-3$ and $6 u-1$. In both cases, the three numbers are pairwise coprime and this proves (26).

It follows from (15), (24) and (26) that

(27) $g_{3}(6 u-5,6 v-1) \leq g_{3}(6 u-5,5)+g_{3}(6 u, 6(v-1)) \leq 3+4(v-1)=4 v-1$.

(16) follows from (22) and (27).

Finally, by (15) we have

$$
g_{3}(6 u, 6 v-1) \leq g_{3}(6 u, 6 v)=4 v .
$$

(17) follows from (22) and (28).

4. In this section we will show that for all $k$ there is an integer $n_{k}$ such that $\lim _{k \rightarrow+\infty}\left(G\left(n_{k}\right)-F\left(n_{k}\right)\right)=+\infty$.

THEOREM 2. There is a positive constant $c_{1}$ and a number $k_{0}$ such that for all $k \geq k_{0}$ there is an integer $n_{k}$ with

$$
G_{k}\left(n_{k}\right)-F_{k}\left(n_{k}\right)>c_{1} k(\log k)^{3}(\log \log k)^{-2} .
$$


PROOF. We need the following result of Erdös :

LEMMA 1. For a certain positive constant $c_{2}$ and all $n \in N$ we can find more than $c_{2} p_{n} \log p_{n}\left(\log \log p_{n}\right)^{-2}$ consecutive integers so that each of them is divisible by at least one of the primes $p_{1}, p_{2}, \cdots, p_{n}$.

In fact, this is Theorem 2 in [1].

By the prime number theorem, $p_{n} \sim n \log n$ so that $p_{n} \log p_{n}\left(\log \log p_{n}\right)^{-2} \sim$ $n(\log n)^{2}(\log \log n)^{-2}$. Thus by Lemma 1 , there is a positive constant $c_{3}$ and for all $k \geq k_{1}$ there are numbers $n_{k}, t_{k} \in N$ so that

$$
n_{k}>c_{3} k(\log k)^{2}(\log \log k)^{-2}
$$

and each of the integers $t_{k}, t_{k}+1, \cdots, t_{k}+n_{k}-1$ is divisible by at least one of the primes $p_{1}, p_{2}, \cdots, p_{k-1}$. Then clearly, the set $\mathcal{A}=\left\{t_{k}, t_{k}+1, \cdots, t_{k}+\right.$ $\left.n_{k}-1\right\}$ has property $P_{k}$ whence

$$
G_{k}\left(n_{k}\right)=g_{k}\left(t_{k}, n_{k}\right)=n_{k}
$$

Now we will give an upper bound for $F_{k}\left(n_{k}\right)$. Assume that $\mathcal{A} \subset\left\{1,2, \cdots, n_{k}\right\}$ and $\mathcal{A}$ has property $\mathbf{P}_{k}$. If $q_{1}<q_{2}<\cdots<q_{l}$ are primes contained in $\mathcal{A}$, then these primes form a subset of $\mathcal{A}$ consisting of pairwise coprime integers. Since $\mathcal{A}$ has property $P_{k}$, this implies that $\ell \leq k-1$, i.e., $\mathcal{A}$ contains at most $k-1$ primes. Thus by (30) and the prime number theorem we have

$$
\begin{aligned}
|\mathcal{A}| & \leq \mid\left\{n: n \leq n_{k}, n \text { is not prime }\right\} \mid+(k-1)= \\
& =n_{k}-\pi\left(n_{k}\right)+(k-1)<n_{k}-c_{4} n_{k}\left(\log n_{k}\right)^{-1}+k-1< \\
& <n_{k}-c_{5} k(\log k)^{3}(\log \log k)^{-2}
\end{aligned}
$$

whence

$$
F_{k}\left(n_{k}\right)<n_{k}-c_{5} k(\log k)^{3}(\log \log k)^{-2} .
$$

(29) follows from (31) and (32), and this completes the proof of Theorem 2. On the other hand, we conjecture that

$$
\lim _{n \rightarrow+\infty} \sup \left(G_{k}\left(n_{k}\right)-F_{k}\left(n_{k}\right)\right)<+\infty
$$


for all $k$ (and, in fact, perhaps the lower bound in (29) is close to the truth). Unfortunately, we have not been able to show this.

Moreover, we conjecture that conjecture (11) can be exterided to the more general function $g_{k}(m, n)$ in the following way : for all $k \geq 2$ and $m, n \in$ $N$, the maximum in (1) is assumed by a set $\mathcal{A} \subset\{m, m+1, \cdots, m+n-1\}$ which consists of the multiples of certain primes $q_{1}, q_{2}, \cdots, q_{k-1}$. However, these primes need not be the first $k-1$ primes, as the following example shows : Let $n=45, n=10, k=4$, and consider

$$
\mathcal{A} \stackrel{\text { def }}{=}\{a: 45 \leq a \leq 54,(a, 2 \cdot 3 \cdot 7)>1\}=\{45 ; 46,48,49,50,51,52,54\} .
$$

Then clearly, $A \in \Gamma_{k}$ so that

$$
g_{4}(45,10) \geq|A|=8 .
$$

On the other hand, we have

$\psi_{3}(45,10)=|\{u: 45 \leq u \leq 54,(u, 2 \cdot 3 \cdot 5)>1\}|=|\{45,46,48,50,51,52,54\}|=7$.

5. In this section, we will estimate the function $h_{(k, \ell)}(m, n)$.

\section{THEOREM 3.}

(i) If $k, \ell, m, n \in N$ and $\ell \geq 2$, then we have

$$
h_{(k, \ell)}(m, n)<(\ell-1)\left(\frac{n}{p_{k+1} P_{k}}+2\right) \prod_{i=1}^{k}\left(p_{i}-1\right) .
$$

(ii) If $k, t, m, n \in \tilde{I}$ and $i \geq \bar{z}$, then we have

$$
h_{(k, \ell)}(m, n) \geq n \prod_{i=1}^{k}\left(1-\frac{1}{p_{i}}\right)\left(1-\prod_{j=k+1}^{k+\ell-1}\left(1-\frac{1}{p_{j}}\right)\right)-P_{k+\ell-1}
$$

Moreover, if $P_{k+2-1} \mid n$, then the term $P_{k+\ell-1}$ on the right hand side can be dropped.

PROOE.

(i) Assume that

$$
\mathcal{A} \subset\{m, m+1, \cdots, m+n-1\}
$$




$$
\left(a, P_{k}\right)=1 \text { for all } a \in \mathcal{A}
$$

and

$$
|\mathcal{A}| \geq(\ell-1)\left(\frac{n}{p_{k+1} P_{K}}+2\right) \prod_{i=1}^{k}\left(p_{i}-1\right)
$$

Let $u$ denote an integer for which $\left|\mathcal{A}\left(P_{k}, u\right)\right|$ is maximal :

$$
\left|\mathcal{A}\left(P_{k}, u\right)\right| \geq\left|\mathcal{A}\left(P_{k}, v\right)\right| \text { for all } v \in \mathbb{Z} \text {. }
$$

By (36) and (37), clearly we have

(38) $\left|\mathcal{A}\left(P_{k}, u\right)\right| \geq \frac{|\mathcal{A}|}{\varphi\left(P_{k}\right)}=|\mathcal{A}| \prod_{i=1}^{k}\left(p_{i}-1\right)^{-1} \geq(\ell-1)\left(\frac{n}{p_{k+1} P_{k}}+2\right)$.

Define the integers $x$ and $y$, respectively, by

$$
x \equiv u\left(\bmod P_{k}\right), x+1 \leq m \leq x+P_{k}
$$

and

$$
x+(y-1) p_{k+1} P_{k}<m+n-1 \leq x+y p_{k+1} P_{K}
$$

so that, by (39) and (40),

$$
y<\frac{m+n-1-x}{p_{k+1} P_{k}}+1 \leq \frac{P_{k}+n-1}{p_{k+1} P_{k}}+1<\frac{n}{p_{k+1} P_{k}}+2 .
$$

For $i \in N$ write

$$
\mathcal{A}=\mathcal{A}_{\left(P_{k}, u\right)} \cap\left\{x+\left((i-1) p_{k+1}+1\right) P_{k}, x+\left((i-1) p_{k+1}+2\right) P_{k}, \cdots, x+i p_{k+1} P_{k}\right\}
$$

Then we have

$$
\mathcal{A}_{\left(P_{k}, u\right)}=U_{i=1}^{y} \mathcal{A}_{i},
$$

thus by (38) and (41) there is an integer $z$ (with $1 \leq z \leq y$ ) such that

$$
\left|\mathcal{A}_{x}\right| \geq \frac{\left|\mathcal{A}_{\left(P_{k}, u\right)}\right|}{y}>(\ell-1)\left(\frac{n}{p_{k+1} P_{k}}+2\right)\left(\frac{n}{p_{k+1} P_{k}}+2\right)^{-1}=\ell-1
$$


whence

$$
\left|\mathcal{A}_{\mathbf{z}}\right| \geq \ell
$$

If $a \in \mathcal{A}_{z}, a^{\prime} \in \mathcal{A}_{z}$ and $a>a^{\prime}$, then $a-a^{\prime}$ is of the form $a-a^{\prime}=j P_{k}$ where $j \in N, j<p_{k+1}$. The prime factors of $j P_{k}$ are smaller, than $p_{k+1}$. It follows that $\left(a, a^{\prime}\right)$ has no prime factor greater than $p_{k}$. On the other hand, by $a \in \mathcal{A}, a^{\prime} \in \mathcal{A}$ we have $\left(a, P_{k}\right)=\left(a^{\prime}, P_{k}\right)=1$, so that $\left(a, a^{\prime}\right)$ has no prime factor not exceeding $p_{k}$. Thus we have $\left(a, a^{\prime}\right)=1$ so that the elements of $\mathcal{A}_{x}$ are pairwise coprime. By (42), it follows that $\mathcal{A}_{x} \subset \mathcal{A}$ cuntains an $\ell$-tuple of pairwise coprime integers so that $\mathcal{A} \notin \Gamma_{\ell}$. This is so for all $\mathcal{A}$ satisfying (35), (36) and (37) which proves (33).

(ii) Define the set $B_{(k, l)}(m, n)$ by

$B_{(k, l)}(m, n)=\left\{b: b \in N, m \leq b<n,\left(b: P_{k}\right)=1,\left(b, p_{k+1} p_{k+2} \cdots p_{k+\ell-1}\right)>1\right\}$

Then clearly, $B_{(k, \ell)}(m, n) \in \Gamma_{\ell}$ and thus

$$
h_{(k, l)}(m, n) \geq\left|B_{(k, l)}(m, n)\right| .
$$

If $P_{k+\ell-1} \mid n$, then by the Chinese remainder theorem we have

$$
\left|B_{(k, \ell)}(m, n)\right|=n \prod_{i=1}^{k}\left(1-\frac{1}{p_{i}}\right)\left(1-\prod_{j=k+1}^{k+\ell-1}\left(1-\frac{1}{p_{j}}\right)\right) \quad\left(\text { for } P_{k+\ell-1} \mid n\right) .
$$

If $P_{k+\ell-1} \mid n$ is not assumed, then define $n^{\prime}$ by $P_{k+\ell-1} \mid, n^{\prime}, n^{\prime} \leq n<$ $n^{\prime}+P_{k+\ell-1}$. Then by (44),

$$
\begin{aligned}
& \left|B_{(k, l)}(m, n)\right| \geq\left|B_{(k, l)}\left(m, n^{\prime}\right)\right|= \\
& \text { (45) } n^{\prime} \prod_{i=1}^{k}\left(1-\frac{1}{p_{i}}\right)\left(1-\prod_{j=k+1}^{k+\ell-1}\left(1-\frac{1}{p_{j}}\right)\right)=n \prod_{i=1}^{k}\left(1-\frac{1}{p_{i}}\right)\left(1-\prod_{j=k+1}^{k+\ell-1}\left(1-\frac{1}{p_{j}}\right)\right)- \\
& -\left(n-n^{\prime}\right) \prod_{i=1}^{k}\left(1-\frac{1}{p_{i}}\right)\left(1-\prod_{j=k+1}^{k+\ell-1}\left(1-\frac{1}{p_{j}}\right)\right) \geq
\end{aligned}
$$




$$
\geq n \prod_{i=1}^{k}\left(1-\frac{1}{p_{i}}\right)\left(1-\prod_{j=h+1}^{k+\ell-1}\left(1-\frac{1}{p_{j}}\right)\right)-P_{k+\ell-1}
$$

The result follows (43), (44) and (45).

6. In this section we will study several consequences of Theorem 3 and, in particular, we will estimate $G_{k}(n)$. First we consider the important special case $\ell=2$, when we get a quite sharp estimate for the function $h_{(k, \ell)}(m, n)$. In fact, in this case the error term is bounded for fixed $k$ :

THEOREM 4. For $k, m, n \in N$ we have

$$
\left|h_{(k, 2)}(m, n)-\frac{n}{p_{k+1}} \prod_{i=1}^{k}\left(1-\frac{1}{p_{i}}\right)\right| \leq P_{k+1} \text {. }
$$

PROOF. By using Theorem 3 with $\ell=2$ we obtain that

$$
\begin{aligned}
& h_{(k, 2)}(m, n)<\left(\frac{n}{p_{k+1} P_{k}}+2\right) \prod_{i=1}^{k}\left(p_{i}-1\right)= \\
& =\frac{n}{p_{k+1}} \prod_{i=1}^{k}\left(1-\frac{1}{p_{i}}\right)+2 \prod_{i=1}^{k}\left(p_{i}-1\right)< \\
& <\frac{n}{p_{k+1}} \prod_{i=1}^{k}\left(1-\frac{1}{p_{i}}\right)+2 P_{k}
\end{aligned}
$$

and

$$
h_{(k, 2)}(m, n) \geq n \prod_{i=1}^{k}\left(1-\frac{1}{p_{i}}\right)\left(1-\left(1-\frac{1}{p_{k+1}}\right)\right)-P_{k+1}=\frac{n}{p_{k+1}} \prod_{i=1}^{k}\left(1-\frac{1}{p_{i}}\right)-P_{k+1}
$$

whence the result follows.

If $\ell>2$ but $\ell$ is not much greater, than $k$, then still we have a satisfactory estimate for $h_{(k, \ell)}(m, n)$ :

THEOREM 5. For all $\varepsilon>0$ there exist numbers $\delta>0, \eta>0$ and $k_{0}(\varepsilon)$ such that for all $k \in N, k>k_{0}(\varepsilon)$ we have

$$
(1-\varepsilon) n\left(\sum_{i=k+1}^{k+\ell-1} \frac{1}{p_{i}}\right) \prod_{i=1}^{k}\left(1-\frac{1}{p_{i}}\right)<h_{(k, \ell)}(m, n)<(1+\varepsilon) \frac{(\ell-1) n}{p_{k+1}} \prod_{i=1}^{k}\left(1-\frac{1}{p_{i}}\right)
$$




$$
\text { for } k>k_{0}(\varepsilon), 2 \leq \ell<k^{1+\delta} \text {, all } m \in N \text { and } n>n_{0}(k, \varepsilon)
$$

$(1-\varepsilon) \frac{(\ell-1) n}{p_{k+1}} \prod_{i=1}^{k}\left(1-\frac{1}{p_{i}}\right)<h_{(k, \ell)}(m, n)<(1+\varepsilon) \frac{(\ell-1) n}{p_{k+1}} \prod_{i=1}^{k}\left(1-\frac{1}{p_{i}}\right)$

$$
\text { for } k>k_{0}(\varepsilon), \ell<\eta k, \text { all } m \in N \text { and } n>n_{0}(k, \varepsilon, \eta)
$$

and

$(1-\varepsilon) \log 2 \frac{n}{\log k} \prod_{i=1}^{k}\left(1-\frac{1}{p_{i}}\right)<h_{(k, k+1)}(m, n)<(1+\varepsilon) \frac{n}{\log k} \prod_{i=1}^{k}\left(1-\frac{1}{p_{i}}\right)$

$$
\text { for } k>k_{0}(\varepsilon) \text {, all } m \in N \text { and } n>n_{0}(k, \varepsilon) \text {. }
$$

PROOF. The upper bound in (46) follows trivially from (33) in Theorem

3. To derive the lower bound in (46) from (34) in Theorem 4, observe that $1-x=e^{-x+O\left(x^{2}\right)}$ and $e^{-x}=1-x+O\left(x^{2}\right)$ for $x \rightarrow 0$, moreover, it follows from

$$
\sum_{p<x} \frac{1}{p}=\log \log x+C+o(1)
$$

and the prime number theorem that if $\rho>0, \delta$ is sufficiently small in terms of $\rho$ and $k>k_{0}(\rho)$, then

$$
\sum_{i=k+1}^{\left[k^{1+\delta}\right]} \frac{1}{p_{i}}<\rho .
$$

(47) follows trivially from (46) and the prime number theorem.

(48) follows from (46), (49) and the prime number theorem and this completes the proof of Theorem 5 .

One may use (48) to estimate $G_{k}(n)$ :

THEOREM 6. For all $\varepsilon>0$ therc is a number $k_{0}=k_{0}(\varepsilon)$ such that for $k>k_{0}(\varepsilon)$, all $m \in N$ and $n>n_{0}(k, \varepsilon)$ we have

(50) $-2^{k} \leq g_{k}(m, n)-\left(1-\prod_{i=1}^{k-1}\left(1-\frac{1}{p_{i}}\right)\right) n<(1+\varepsilon) \frac{n}{\log k} \prod_{i=1}^{k-1}\left(1-\frac{1}{p_{i}}\right)$. 
Moreover, for $P_{k-1} \mid n$ the lower bound can be replaced by 0 .

Note that since except for $g_{k}(m, n)$, all the terms in (50) are independent of $m$, it follows that

COROLLARY 2. For $\varepsilon>0, k>k_{0}(\varepsilon)$ and $n>n_{0}(k, \varepsilon)$ we have

$$
-2^{k}<G_{k}(n)-\left(1-\prod_{i=i}^{h-1}\left(1-\frac{1}{p_{i}}\right)\right) n<(1+\varepsilon) \frac{n}{\log k} \prod_{i=1}^{k-1}\left(1-\frac{1}{p_{i}}\right)
$$

where the lower bound can be replaced by 0 for $\boldsymbol{P}_{\boldsymbol{k}} \mid \boldsymbol{n}$.

PROOF OF THEOREM 6. It follows from (3) and (6) that

$$
\begin{aligned}
g_{k}(m, n) & -\left(1-\prod_{i=1}^{k-1}\left(1-\frac{1}{p_{i}}\right)\right) n \geq \psi_{k-1}(m, n)- \\
& -\left(1-\prod_{i=1}^{k-1}\left(1-\frac{1}{p_{i}}\right)\right) n \geq-2^{k}
\end{aligned}
$$

and, by (7), this lower bound can be replaced by 0 for $P_{k-1} \mid n$.

On the other hand, assume that $\mathcal{A} \subset\{m, m+1, \cdots, m+n-1\}$ and $A \in \Gamma_{k}$. Then by (6) and (48), for $k>k_{0}(\varepsilon)$, all $m \in N$ and $n>n_{0}(k, \varepsilon)$ we have

$$
\begin{aligned}
& |\mathcal{A}|=\left|\left\{a: a \in \mathcal{A},\left(a, P_{k-1}\right)>1\right\}\right|+\left|\left\{a: a \in \mathcal{A},\left(a, P_{k-1}\right)=1\right\}\right| \leq \\
& \leq\left|\left\{a: m \leq a<m+n,\left(a, P_{k-1}\right)>1\right\}\right|+h_{(k-1, k)}(m, n)= \\
(52) & =\psi_{k-1}(m, n)+h_{(k-1, k)}(m, n)< \\
& <\left(\left(1-\prod_{i=1}^{k-1}\left(1-\frac{1}{p_{i}}\right)\right) n+2^{k}\right)+\left(1+\frac{\varepsilon}{2}\right) \frac{n}{\log (k-1)} \prod_{i=1}^{k-1}\left(1-\frac{1}{p_{i}}\right)< \\
& <\left(1-\prod_{i=1}^{k-1}\left(1-\frac{1}{p_{i}}\right)\right) n+(1+\varepsilon)_{\frac{n}{\log k} \prod_{i=1}^{k-1}}\left(1-\frac{1}{p_{i}}\right) .
\end{aligned}
$$

The result follows from (51) and (52). 
7. In this section, we will generalize Theorem 3 in [3] and Theorem 8 in [4]. For $k \in N, k \geq 2$ we write

$$
\alpha_{k}=\inf _{n \in N} \frac{G_{k}(n)}{n}
$$

(It is easy to see that $\alpha_{2}=1 / 2, \alpha_{3}=2 / 3$ ).

THEOREM 7. Let $k \in N, k \geq 2$ and $\varepsilon>0$. There are numbers $n_{0}=$ $n_{0}(k, \varepsilon)$ and $c_{6}=c_{6}(k, \varepsilon)$ such that if $n>n_{0}, m \in N, \mathcal{A} \subset\{m, i n+1, \cdots, m+$ $n-1\}$ and

$$
|\mathcal{A}|>\left(\alpha_{k}+\varepsilon\right) n
$$

then

$$
\Phi_{k}(\mathcal{A})>c_{6} n^{k}
$$

Note that it follows trivially from Theorem 7 that for all $k \geq 2, \lim _{n \rightarrow+\infty} \frac{G_{k}(n)}{n}$ exists (and this limit is equal to $\alpha_{k}$ ).

PROOF. The proof will be based on the following lemma :

LEMMA 2. To every $\rho>0$ and $\delta>0$ there is an $r_{0}=r_{0}(\rho, \delta)$ so that if $r \geq r_{0}, n>n_{1}(\rho, \delta, r), m \in N$ and $u=1,2, \ldots, P_{r}$, then for all but $\rho \frac{n}{P_{r}}$ integers $b$ satisfying

$$
m \leq b<m+n, \quad b \equiv u\left(\bmod P_{r}\right)
$$

we have

$$
\gamma(b) \stackrel{\text { def }}{=} \sum_{p \mid b, p_{r}<p<n} \frac{1}{p}<\delta .
$$

PROOF. Denote the set of the integers satisfying (55) by $B$ and write $B_{1}=\{b: b \in B, \gamma(b) \geq \delta\}$. By the Chinese remainder theorem, $\sum_{i=1}^{+\infty} \frac{1}{p_{i}^{2}}<+\infty$ and $\sum_{p<n} \frac{1}{p} \sim \log \log n$, for $r>r_{0}(\rho, \delta)$ and $n>n_{1}(\rho, \delta, r)$ we have 


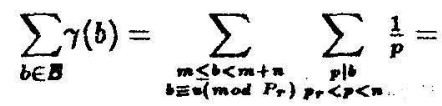

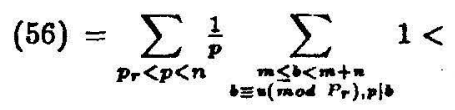

$$
\begin{aligned}
& <\sum_{p_{r}<p<n} \frac{1}{p}\left(\frac{n}{p P_{r}}+1\right)<\frac{n}{P_{r}} \sum_{p_{r}<p} \frac{1}{p^{2}}+\sum_{p<n} \frac{1}{p}< \\
& <\frac{\rho \delta}{2} \cdot \frac{n}{P_{r}}+2 \log \log n<\rho \delta \frac{n}{P_{r}} \text {. }
\end{aligned}
$$

On the other hand, clearly we have

$$
\sum_{b \in \boldsymbol{B}} \gamma(b) \geq \sum_{b \in \mathbb{B}_{1}} \gamma(b) \geq \sum_{b \in \mathbb{B}} \delta=\delta\left|B_{1}\right| .
$$

It follows from (56) and (57) that

$$
\left|B_{1}\right|<\rho \frac{n}{P_{r}}
$$

and this completes the proof of Lemma 2.

Now we prove Theorem 7. By the definition of $\alpha_{k}$, there is a positive integer $n_{1}$ such that

$$
G_{k}\left(n_{1}\right)<\left(\alpha_{k}+\frac{\varepsilon}{4}\right) n_{1}
$$

so that

$$
g_{k}\left(m, n_{1}\right)<\left(\alpha_{k}+\frac{\varepsilon}{4}\right) n_{1} \text { for all } m \in N
$$

Let $r$ be a positive integer such that

$$
r>\max \left(n_{1}, r_{0}\left(\frac{\varepsilon}{8 n_{1}}, \frac{\varepsilon}{32 k}\right)\right)
$$

and write $n_{1} P_{r}=M$.

By (53) we have

$$
P_{r} \max _{0 \leq i<P_{r}} \sum_{j=1}^{n_{1}}\left|A_{\left(j r_{i n}+j\right)}\right| \geq
$$




$$
\geq \sum_{i=0}^{P_{r}-1} \sum_{j=1}^{n_{1}}\left|\mathcal{A}_{\left(M, \pi_{1}+j\right)}\right|=\sum_{u=1}^{M^{T}}\left|\mathcal{A}_{(M, u)}\right|=|\mathcal{A}|>\left(a_{k}+\varepsilon\right) n .
$$

It follows that there is an integer $i$ such that $0 \leq i<P_{r}$ and

$$
\sum_{j=1}^{n_{1}}\left|\mathcal{A}_{\left(M, \mathrm{in}_{1}+j\right)}\right|>\left(\alpha_{k}+\varepsilon\right) \frac{n}{P_{r}}
$$

Clearly, for all $u \in \mathscr{Z}$ we have

$$
\left|\mathcal{A}_{(M, u)}\right| \leq|\{a: m \leq a<m+n, a \equiv u(\bmod M)\}|<\frac{n}{M}+1 .
$$

(60) and (61) imply that for sufficiently large $n$ there exist integers $j_{1}, j_{2}, \cdots, j_{t}$ such that

$$
\begin{aligned}
1 \leq j_{1} & \leq j_{2} \leq \cdots<j_{t} \leq n_{1}, \\
t & \geq\left(\alpha_{k}+\frac{\varepsilon}{2}\right) n_{1}
\end{aligned}
$$

and

$$
\left|\mathcal{A}_{\left(M, i_{1}+j_{u}\right)}\right|>\frac{\varepsilon}{4} \frac{n}{M} \text { for } u=1,2, \cdots, t
$$

since otherwise, writing $\mathcal{J}_{1}=\left\{j: 1 \leq j \leq n_{1},\left|\mathcal{A}_{\left(M, \text { in }_{1}+j\right)}\right|>\frac{\varepsilon}{4} \frac{n}{M}\right\}$ and $\mathcal{J}_{2}=\left\{j: 1 \leq j \leq n_{1},\left|\mathcal{A}_{\left(M, n_{1}+j\right)}\right| \leq \frac{\varepsilon}{i} \frac{n}{M}\right\}$ by (61) for large $n$ we had

$$
\begin{aligned}
& \sum_{j=1}^{n_{1}}\left|\mathcal{A}_{\left(M, n_{1}+j\right)}\right|=\sum_{j \in \mathcal{J}_{i}}\left|\mathcal{A}_{\left(M, n_{1}+j\right)}\right|+\sum_{j \in J_{2}}\left|\mathcal{A}_{\left(M, i n_{1}+j\right)}\right| \leq \\
& \leq \sum_{j \in J_{1}}\left(\frac{n}{M}+1\right)+\sum_{j \in J_{2}} \frac{\varepsilon}{4} \frac{n^{M}}{M}=\left(\frac{n}{M}+1\right)\left|\mathcal{J}_{1}\right|+\frac{\varepsilon}{4} \frac{n}{M}\left|\mathcal{J}_{2}\right|< \\
& \left(\frac{n}{M}+1\right)\left(\alpha_{k}+\frac{\varepsilon}{4}\right) n_{i}+\frac{\varepsilon}{4} \frac{n}{M} n_{1}= \\
& =\left(\alpha_{k}+\frac{\varepsilon}{2}\right) \frac{n}{P_{r}}+\left(\alpha_{k}+\frac{\varepsilon}{4}\right) n_{1}<\left(\alpha_{k}+\varepsilon\right) \frac{n}{P_{r}}
\end{aligned}
$$


and this contradicts $(60)$.

By (58), (62) and (63), the set $\left\{i n_{1}+j_{1}, i n_{1}+j_{2}, \cdots, i n_{1}+j_{t}\right\}$ contains a subset $\left\{v_{1}, v_{2}, \cdots, v_{k}\right\}$ consisting of $k$ pairwise coprime integers. Then we have

$$
\begin{gathered}
v_{x} \not \equiv v_{y}(\bmod M) \text { for } 1 \leq x<y \leq k \\
\left(v_{x}, v_{y}\right)=1 \text { for } 1 \leq x<y \leq k
\end{gathered}
$$

and, by (64),

$$
\left|\mathcal{A}_{\left(M, v_{\varepsilon}\right)}\right|>\frac{\varepsilon}{4} \frac{n}{M} \text { for } x=1,2, \cdots, k .
$$

Now we will show that it suffices to prove

LEMMA 3. Using the notations above and writing $\mathcal{D}=\{d: m \leq d<$ $\left.m+n, \gamma(d)=\sum_{p \mid d, p_{r}<p<n} \frac{1}{p}<\frac{\epsilon}{32 k}\right\}$,

(i) there are more than $\frac{\varepsilon}{8} \frac{n}{m}$ integers $d_{1}$, satisfying $d_{1} \in D \cap \mathcal{A}_{\left(M, v_{1}\right)}$,

(ii) if $j \in\{2,3, \cdots, k\}$ and $d_{1}, \cdots, d_{j-1}$ are integers with $d_{1} \in \mathcal{D} \cap \mathcal{A}_{\left(M, V_{1}\right)}, \cdots, d_{j-1} \in$ $\mathcal{D} \cap A_{\left(M v_{j-1}\right)}$ and $($ for $j \geq 3)$

$$
\left(d_{x}, d_{y}\right)=1 \text { for } 1 \leq x<y \leq j-1 \text {, }
$$

then there are more than $\frac{\epsilon}{16} \stackrel{n}{M}$ integers $d_{j}$ satisfying $d_{j} \in \mathcal{D} \cap \mathcal{A}_{\left(M, V_{j}\right)}$ and

$$
\left(d_{x}, d_{j}\right)=1 \text { for } 1 \leq x \leq j-1
$$

Assume namely that Lemma 3 has been proved. Select a $d_{1}$ in the way described in (i). Then select $d_{2}, \cdots, d_{k}$ successively in the way described in (ii). In this way, we obtain distinct $k$-tuples $\left(d_{1}, d_{2}, \cdots, d_{k}\right)$ all whose elements belong to $\mathcal{A}$ and whose elements are pairwise coprime. Thus $\Psi_{k}(\mathcal{A})$ is greater than or equal to the number of these $k$-tuples $\left(d_{1}, \cdots, d_{k}\right)$. To give a lower bound for the number of these $k$-tuples, observe that by (i), $d_{1}$ can be chosen in more than $\frac{\varepsilon}{8} \frac{n}{M}$ ways; if $d_{1}$ is given, then by (ii), $d_{2}$ can be chosen 
in more, than $\frac{\epsilon}{16} \frac{n}{M}$ ways (independently of $d_{1}$ ), etc., finally, if $d_{1}, \cdots, d_{k-1}$ are given, then $d_{k}$ can be chosen in more, than $\frac{\varepsilon}{16} \frac{n}{m}$ ways. Thus the total number of these $k$-tuples $\left(d_{1}, \cdots, d_{k}\right)$ is greater, than $\frac{c}{8} \frac{n}{M} \cdot \frac{c}{16} \frac{n}{M} \cdots \cdots \frac{\varepsilon}{16} \frac{n}{M}$ so that

$$
\phi_{k}(\mathcal{A})>\frac{2 \varepsilon^{k}}{16^{k} M^{k}} n^{k}>c_{7}(k, \varepsilon) n^{k}
$$

and this proves (54).

It remains to prove Lemma 3.

PROOF OF LEMMA 3. By Lemma 2 (with $\rho=\frac{\varepsilon}{8 n_{1}}$ and $\delta=\frac{\varepsilon}{32 k}$ ) (59) and (67) for $1 \leq x \leq k$ we have

$$
\begin{aligned}
& \left|\mathcal{D} \cap \mathcal{A}_{\left(M, V_{x}\right)}\right|=\left|\mathcal{A}_{\left(M, V_{x}\right)}\right|-\left|\left\{a: a \in \mathcal{A}_{\left(M, V_{x}\right)} a \notin \mathcal{D}\right\}\right|> \\
& >\frac{\varepsilon}{4} \frac{n}{M}-\left|\left\{a: a \equiv v_{x}(\bmod M), m \leq a<m+n, \gamma(a) \geq \frac{\varepsilon}{32 k}\right\}\right| \geq \\
(69) & \geq \frac{\varepsilon}{4} \frac{n}{M}-\left|\left\{a: a \equiv v_{x}\left(\bmod P_{r}\right), m \leq a<m+n, \gamma(a) \geq \frac{\varepsilon}{32 k}\right\}\right| \geq \\
& \geq \frac{\varepsilon}{4} \frac{n}{M}-\frac{\varepsilon}{8 n_{1}} \frac{n}{P_{r}}=\frac{\varepsilon}{8} \frac{n}{M}
\end{aligned}
$$

which, with $x=1$, proves (i).

Assume now that $2 \leq j \leq k$ and $d_{1}, \cdots, d_{j-1}$ are given as described in (ii). Then clearly,

$$
\left|\left\{d: d \in \mathcal{D} \cap \mathcal{A}_{\left(M, V_{j}\right)},\left(d_{1}, d\right)=\cdots=\left(d_{j-1}, d\right)=1\right\}\right| \geq
$$

$$
\geq\left|D \cap \mathcal{A}_{\left(M, V_{j}\right)}\right|-\sum_{i=1}^{j-1}\left|\left\{d: d \in D \cap \mathcal{A}_{\left(M, V_{j}\right)},\left(d_{i}, d\right)>1\right\}\right|
$$

Assume that $1 \leq i \leq j-1, d \in D \cap \mathcal{A}_{\left(M, V_{j}\right)}$ and $p$ is a prime with

$$
p \mid\left(d_{i}, d\right) \text {. }
$$

By $m \leq d_{i}, d<m+n$, we have

$$
\left|d_{i}-d\right|<n
$$


Moreover, by $d_{i} \in \mathcal{A}_{\left(M, V_{i}\right)}, d \in \mathcal{A}_{\left(M, V_{j}\right)}, i<j$ and (65) we have

$$
d_{i} \neq d \text {. }
$$

It follows from (71), (72) and (73) that $p<n$.

By $d_{i} \in \mathcal{A}_{\left(M, V_{i}\right)}, d \in \mathcal{A}_{\left(M, V_{j}\right)}$ we have

$$
d \equiv v_{i}(\bmod M), \quad d \equiv v_{j}(\bmod M)
$$

If $p \leq p_{r}$, then it follows from (71), (74) and $p \mid M=n_{1} P_{r}$ that

$$
v_{i} \equiv v_{j} \equiv 0(\bmod p)
$$

so that $p \mid\left(v_{i}, v_{j}\right)$ with $i<j$ which contradicts (66).

Thus (71) implies that $p_{r}<p<n$ and thus, by (59), $(p, M)=1$ so that, by $d_{i} \in \mathcal{D}$, for $1 \leq i \leq j-1$ we have

$$
\begin{aligned}
& \left|\left\{d: d \in \mathcal{D} \cap \mathcal{A}_{\left(M, V_{j}\right)},\left(d_{i}, d\right)>1\right\}\right| \leq \\
& \left.\leq \sum_{p \mid d_{i}, p_{r}<p<n}\left|\left\{d: m \leq d<m+n, d \equiv v_{j}(\bmod M)\right\}, p\right| d\right\} \mid< \\
(75) & <\sum_{p \mid d_{i}, p_{r}<p<n}\left(\frac{n}{p M}+1\right)= \\
& =\frac{n}{M} \sum_{p \mid d_{i}, p_{r}<p<n} \frac{1}{p}+\left|\left\{p: p \mid d_{i}, p_{r}<p<n\right\}\right|< \\
& <\frac{\varepsilon}{32 k} \frac{n}{M}+\left|\left\{p: p \mid d_{i}, p_{r}<p<n\right\}\right| .
\end{aligned}
$$

By (68), it follows (69) (with $x=j$ ), (70) and (75) that for large $n$ we have

$$
\begin{aligned}
& \left|\left\{d: d \in \mathcal{D} \cap \mathcal{A}_{\left(M, V_{j}\right)},\left(d_{1}, d\right)=\cdots=\left(d_{j-1}, d\right)=1\right\}\right| \geq \\
& \geq \frac{\varepsilon}{8} \frac{n}{M}-\sum_{i=1}^{j-1}\left(\frac{\varepsilon}{32 k} \frac{n}{M}+\left|\left\{p: p \mid d_{i}, p_{r}<p<n\right\}\right|\right)= \\
& =\frac{\varepsilon}{8} \frac{n}{M}-(j-1) \frac{\varepsilon}{32 k} \frac{n}{M}-\sum_{i=1}^{j-1}\left|\left\{p: p \mid d_{i}, p_{r}<p<n\right\}\right|> \\
& >\frac{\varepsilon}{8} \frac{n}{M}-\frac{\varepsilon}{32} \frac{n}{M}-\pi(n)>\frac{\varepsilon}{16} \frac{n}{M}
\end{aligned}
$$

which proves (ii) and this completes the proof of Lemma 3. 


\section{References}

[1] P. Erdös, On the difference of consecutive primes, Quart. J. Oxford 6 (1935), 124-128.

[2] P. Erdös, Remarks in number theory, IV (in Hungarian), Mat. Lapok 13 (1962), 228-255.

[3] P. Erdös, A. Sárközy and E. Szemerédi, On some extremal properties of sequences of integers, Annales Univ. Sci. Budapest, Eötvös 12 (1969), 131-135.

[4] P. Erdös, A. Sárközy and E. Szemerédi, On some extremal properties of sequences of integers, II, Publicationes Math. Debrecen 27 (1980), 117-125.

[5] C. Szab6 and G. Toth, Maximal sequences not containing 4 pairwise coprime integers (in Hungarian), Mat. Lapok 32 (1985), 253-257. 